\title{
Investigating a multigene prognostic assay based on significant pathways for Luminal A breast cancer through gene expression profile analysis
}

\author{
HAIYAN GAO, MEI YANG and XIAOLAN ZHANG \\ Department of Breast Surgery, Changzhou No. 2 People's Hospital, \\ Affiliated to Nanjing Medical University, Changzhou, Jiangsu 213000, P.R. China
}

Received June 22, 2017; Accepted January 22, 2018

DOI: $10.3892 / \mathrm{ol} .2018 .7940$

\begin{abstract}
The present study aimed to investigate potential recurrence-risk biomarkers based on significant pathways for Luminal A breast cancer through gene expression profile analysis. Initially, the gene expression profiles of Luminal A breast cancer patients were downloaded from The Cancer Genome Atlas database. The differentially expressed genes (DEGs) were identified using a Limma package and the hierarchical clustering analysis was conducted for the DEGs. In addition, the functional pathways were screened using Kyoto Encyclopedia of Genes and Genomes pathway enrichment analyses and rank ratio calculation. The multigene prognostic assay was exploited based on the statistically significant pathways and its prognostic function was tested using train set and verified using the gene expression data and survival data of Luminal A breast cancer patients downloaded from the Gene Expression Omnibus. A total of 300 DEGs were identified between good and poor outcome groups, including 176 upregulated genes and 124 downregulated genes. The DEGs may be used to effectively distinguish Luminal A samples with different prognoses verified by hierarchical clustering analysis. There were 9 pathways screened as significant pathways and a total of 18 DEGs involved in these 9 pathways were identified as prognostic biomarkers. According to the survival analysis and receiver operating characteristic curve, the obtained 18-gene prognostic assay exhibited good prognostic function with high sensitivity and specificity to both the train and test samples. In conclusion the 18-gene prognostic assay including the key genes, transcription factor 7-like 2, anterior parietal cortex and lymphocyte
\end{abstract}

Correspondence to: Dr Haiyan Gao, Department of Breast Surgery, Changzhou No. 2 People's Hospital, Affiliated to Nanjing Medical University, 68 Gehu Road, Changzhou, Jiangsu 213000, P.R. China

E-mail: dsjhsdhwwiiwi@qq.com

Key words: Luminal A breast cancer, differentially expressed genes, significant pathways, multigene prognostic assay enhancer factor-1 may provide a new method for predicting outcomes and may be conducive to the promotion of precision medicine for Luminal A breast cancer.

\section{Introduction}

Breast cancer is the most commonly diagnosed cancer in women and approximately accounts for $29 \%$ of all new cancers in women, and it is the leading cause of cancer-related death in women worldwide (1). Breast cancer is considered as a heterogeneous disease but not a single disease at molecular and clinical levels $(2,3)$. The well-known characteristics of breast cancer-associated factors include pathologic and clinical characteristics of the primary tumor, tumor histology, axillary lymph node (ALN) status, estrogen receptor (ER) content, progesterone receptor (PR) content, content, tumor HER 2 status, detectable metastatic disease, patient age, patient comorbid conditions, and menopausal status $(3,4)$. Based on the determination of ER, PR, HER2, and Ki-67, breast cancer is often to be accepted as four subtypes, namely Luminal A, Luminal B, Erb-B2 overexpression and Basal-like (also known as Triple negative breast cancer) according to St Gallen (3). Luminal A (ER positive, PR positive, HER2/neu negative) is the most common subtype, accounting for more than $50 \%$ of all breast cancer patients $(5,6)$.

Multigene predictors have been introduced by various technologies, including immunohistochemistry (IHC), reverse transcription-quantitative polymerase chain reaction (RT-qPCR), fluorescence in situ hybridization (FISH) and genomic microarrays (7). Nowadays, some microarray-based multigene predictors have been developed as predictors of response to hormonal therapy $(8,9)$, predictors of response to multiagent cytotoxic chemotherapy (10-13) and independent prognostic biomarkers (14-16).

Studies have shown that the recurrence score based on a 21-gene assay is a recurrence predictor for breast cancer patients receiving adjuvant endocrine therapy (17-19). Recurrence score is an independent predict factor for the response to adjuvant chemotherapy (20,21). Patients with high scores could benefit from adjuvant treatments, whereas those with low scores could not regardless of the pathologic and clinical characteristics. In ATAC trial (22), the risk of recurrence score obtained using a 
50-gene assay was seen to have an obvious relationship with the 10 years distant recurrence risk in postmenopausal breast cancer women treated with tamoxifen or aromatase inhibitors. In addition, a first commercialized microarray-based multigene assay containing 70 genes, primarily associated with proliferation, metastasis, invasion, stromal integrity, and angiogenesis, is approved by the FDA's new in diagnostic multivariate index assay classification (7).

Though multigene predictors have been widely investigated and used for the breast cancer, there are still rare studies focusing on the prognosis of subtypes. According to retrospective analyses and authoritative guidelines, these subtypes are associated with different relapse-free survival and overall survival and the patients with different subtypes should be administrated with different systemic treatment strategies (3). In this study, we aimed to utilize microarray profiling to investigate potential biomarkers that are differentially expressed in women with Luminal A-like breast cancer based on significant pathways analysis through gene expression profiles analysis. To validate the ability of the candidate multigene assay for the prediction of clinical outcomes, the gene expression data and survival data of Luminal A breast cancer patients were downloaded from Gene Expression Omnibus for analysis.

\section{Materials and methods}

Gene expression data. The gene expression profiles of breast cancer patients were downloaded from The Cancer Genome Atlas (TCGA, https://cancergenome.nih.gov/) database with the deadline of December 27, 2016, including 20,501 genes obtained from 1,160 samples (1,041 tumor tissue samples, 112 normal tissue samples and 7 peripheral blood samples). According to the clinical information of ER, PR and HER2 information (23), 370 Luminal A breast cancer samples were screened.

Data processing and differentially expressed genes (DEGs) identifying. The expression profile data of Luminal A patients were normalized. Z-score correction method was utilized to rule out the difference at gene expression level (24). A total of 249 Luminal A samples from alive patients were assigned as good outcome group; whereas a total of 47 Luminal A samples from dead patients were assigned as poor outcome group. $\mathrm{P}<0.01$ and $\mid \log _{2}$ Fold-Change $(\mathrm{FC}) \mid>1$ were regarded as the cut-off criteria to screen out DEGs between the good and poor outcome groups using LIMMA package (25).

Hierarchical clustering analysis. Hierarchical clustering analysis was conducted for the DEGs using heatmap2 package in $\mathrm{R}$ language (26) and the result was visualized using the form of heatmap.

Identifying statistically significant pathways. The pathway information were download from Kyoto Encyclopedia of Genes and Genomes (KEGG) (http://www.kegg.jp/kegg/pathway. html) database on March 1, 2017. The KEGG pathway enrichment analyses were performed based on pathway feature vector calculation (27) and nearest shrunken centroids (28). Briefly, the KEGG pathway was scored using the expression values of the DEGs in all samples. The sample was projected by taking

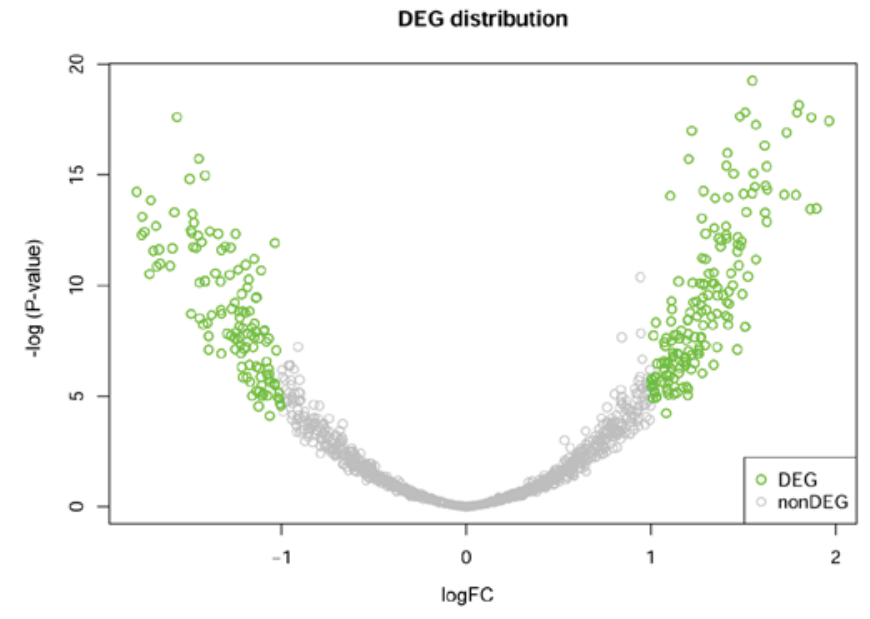

Figure 1. The volcanic map of 300 DEGs. The abscissa represents $\log _{2} \mathrm{FC}$ and the ordinate represents the negative logarithm of P-value. DEG, differentially expressed genes; FC, fold-change.

the upregulated score and downregulated score as coordinates. The accuracy of the good group and the poor group was evaluated by calculating the geometric center of the same sample and specifying the radius (27). The pathways with accuracy more than $80 \%$ in these two groups were screened. The statistically significant pathways were recognized by calculating the Ratio of rank and converting to P-value according to the random 10,000 times perturbation of the background library (train set samples) (28).

Identifying prognostic biomarkers and training. Survival analysis for Luminal A breast cancer samples in TCGA were performed using DEGs involved in the obtained significant pathways (29). The support vector machine (SVM) classification model was constructed using these DEGs. Meanwhile, the model was trained using the Luminal A samples and the receiver operating characteristic (ROC) curve was drawn.

Verification of multigene prognostic assay. The reliability and repeatability of the multigene assay were verified using the gene expression profiles of GSE2034 (https://www.ncbi.nlm. nih.gov/geo/geo2r/?acc=GSE2034) and the survival data of Luminal A breast cancer patients was downloaded from Gene Expression Omnibus (GEO, http://www.ncbi.nlm.nih.gov/geo) database. Besides, the SVM model was also utilized to test the multigene assay, and the accuracy of the model was analyzed using the ROC curve.

\section{Results}

DEGs for Luminal A breast cancer with good and poor outcome groups. Using LIMMA package, a total of 300 DEGs were identified between 249 samples from good outcome group and 47 samples from poor outcome group, including 176 upregulated genes and 124 downregulated genes (Fig. 1). It can be observed from the figure that the data are homogenized to eliminate the deviation and the deviation scores of most genes were concentrated in -1 to 1 . The genes distributed in the two branches were the most significant DEGs. 


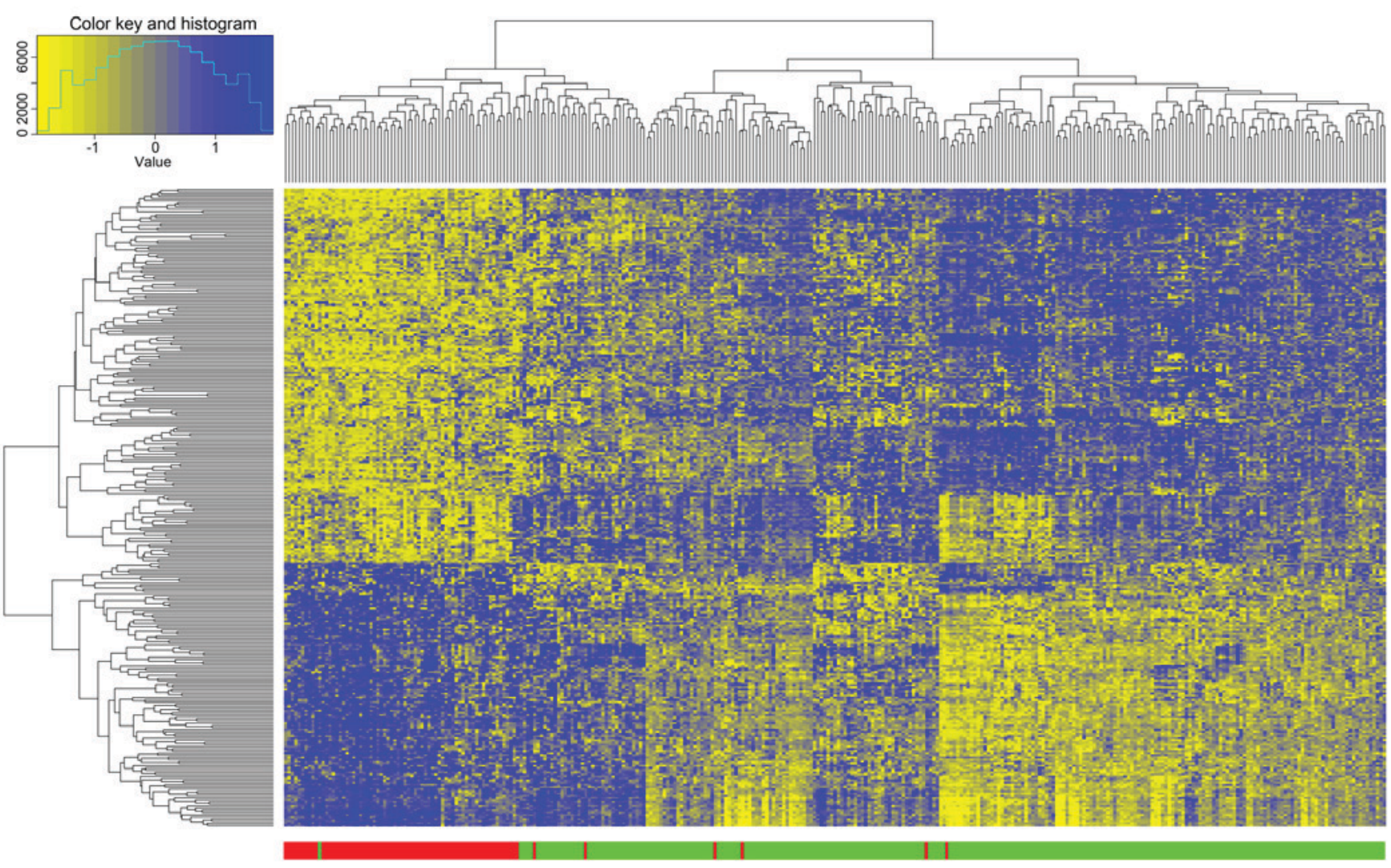

Figure 2. The heat map of 300 DEGs in the dead and alive samples. The abscissa represents the expression value of 300 DEGs in all samples and the ordinate represents the two sets of samples. Blue represents high expression, and yellow represents low expression. Meanwhile, the dead and alive samples were marked with red and green labels, respectively. DEG, differentially expressed genes.

Clustering of DEGs. The 300 DEGs identified between good and poor outcome groups were selected for hierarchical clustering analysis. As presented in Fig. 2, 68 of 74 dead samples were clustered together and only 6 samples were clustered to the alive group with a precision of $92 \%$. Meanwhile, 248 of 249 alive samples were clustered together and only 1 sample was clustered to the dead group with a precision of $99.6 \%$. This result indicated that the DEGs could be used to effectively distinguish Luminal A samples with different prognoses.

Statistically significant pathways. Total 9 pathways with accuracy more than $80 \%$ in the two groups were screened. The significance analysis for these 9 pathways was conducted using Nearest Shrunken Centroids and the results are shown in Table I. It was observed that all of the 9 pathways significantly distinguished cancer samples with good outcome from cancer samples with poor outcome $(\mathrm{P}<0.05)$.

Identifying prognostic biomarkers based on the significant pathways. The DEGs involved in these 9 significant pathways were collected and a total of 18 DEGs were identified as prognostic biomarkers (Table II). Three genes [transcription factor 7-like 2 (TCF7L2), anterior parietal cortex (APC), and lymphocyte enhancer factor-1 (LEFI)], were involved in four pathways, one gene [cyclin E1 (CCNE1)] in three pathways, four [S-phase kinase-associated protein 2 (SKP2), human frizzled-7 (FZD7), polo-like kinase 1 ( $P L K 1)$, and B cell lymphoma 2 (BCL2)] in two pathways and ten [proteasome activator subunit 4 (PSME4), prenyldiphosphate synthase, subunit 1 ( $P D S S 1)$, promoters for human DNA-PK cs $(P R K D C)$, TTK protein kinase $(T T K)$, minichromosome maintenance deficient 4 (MCM4), progesterone receptor $(P G R)$, proteasome subunit alpha 7 (PSMA7), MDM2 proto-oncogene (MDM2), laminin subunit beta 2 (LAMB2), and proteasome 26S subunit, non-ATPase 7 (PSMD7)] in one pathway. Meanwhile, the annotation results for the 18 genes based on the TCGA database are shown in Fig. 3A. Simultaneously, the heat map was shown for the changes of the 18 DEGs in TCGA breast cancer samples (Fig. 3B). It can be seen that the expression of these 18 DEGs in TCGA breast cancer samples are almost upregulated.

Survival analysis for the 18 DEGs in train set. The survival analysis was conducted for the Luminal A breast patient samples with abnormal expression of these 18 genes and samples with normal expression of these 18 genes in TCGA database. As a result, the samples with abnormal expression of these 18 genes showed a significantly lower survival rate than samples with normal expression of these 18 genes (Fig. 4A, $\mathrm{P}=0.0319$ ).

Additionally, the average AUC (area under curve) was 0.871 for the ROC of these 18 genes calculated from the random train set in TCGA database. The sensitivity was 0.913 and the specificity was 0.88 (Fig. 4B).

The verification of multigene prognostic assay in test set. The multigene assay was verified using the Luminal A 
Table I. Nine significant pathways according to Kyoto Encyclopedia of Genes and Genomes analysis nearest shrunken centroids.

\begin{tabular}{lccc}
\hline Pathway & $\begin{array}{c}\text { Initial } \\
\text { precision }\end{array}$ & $\begin{array}{c}\text { Average } \\
\text { value }\end{array}$ & P-value \\
\hline Colorectal cancer & 0.7814 & 0.5775 & $1.27 \mathrm{E}-12$ \\
Oocyte meiosis & 0.8050 & 0.6291 & $9.24 \mathrm{E}-09$ \\
Wnt signaling pathway & 0.7033 & 0.5088 & $3.38 \mathrm{E}-06$ \\
Terpenoid backbone & 0.6268 & 0.4298 & $5.80 \mathrm{E}-04$ \\
biosynthesis & & & \\
Endometrial cancer & 0.5582 & 0.4769 & $7.90 \mathrm{E}-04$ \\
Cell cycle & 0.5843 & 0.4648 & $2.81 \mathrm{E}-03$ \\
Proteasome & 0.5422 & 0.4178 & $1.13 \mathrm{E}-02$ \\
Basal cell carcinoma & 0.5715 & 0.4104 & $1.50 \mathrm{E}-02$ \\
Small cell lung cancer & 0.5567 & 0.3995 & $1.72 \mathrm{E}-02$ \\
\hline
\end{tabular}

The average value column represents the average score of 100 iterations.

breast patient samples with abnormal expression of these 18 genes and samples with normal expression of these 18 genes obtained from GSE2034 database. As a result, the samples with abnormal expression of these 18 genes showed a significantly lower survival rate than samples with normal expression of these 18 genes (Fig. 5A, P=0.0279). According to the ROC for the test set, the average AUC was 0.793 with sensitivity of 0.832 and specificity of 0.779 (Fig. 5B).

\section{Discussion}

With the promotion and put forward of precision medical, studies focusing on special subtypes of breast cancer are particularly meaningful. Regardless of the development of the multigene prognostic assay for breast cancer, our study still has a critical necessary for the prognostic study of breast cancer by focusing on the Luminal A subtype. According to our results, a total of 300 DEGs were identified between good prognosis group and poor prognosis group, including 176 upregulated genes and 124 downregulated genes. Based on the hierarchical clustering analysis, these DEGs could clearly distinguish the samples of the two groups. Meanwhile, the 18 genes predictors were involved in 9 significant pathways, including cancer-related pathways (colorectal cancer, endometrial cancer, basal cell carcinoma and small cell lung cancer), oocyte meiosis, Wnt signaling pathway, Terpenoid backbone biosynthesis, cell cycle and proteasome were selected. According to the survival analysis and ROC curve, the obtained 18-gene prognostic assay exhibited a good prognostic function with high sensitivity and specificity for the train set samples and verification set samples.

TCF7L2 was identified as a key gene in the multigene prognostic assay and it was involved in four significant pathways, namely Wnt signaling pathway, colorectal cancer, endometrial cancer and basal cell carcinoma, based on the pathway analysis. TCF7L2, located on chromosome 10q25.2, plays a critical role in cancer cell growth, apoptosis, invasion
Table II. DEGs identified as prognostic biomarkers based on the significant pathways.

\begin{tabular}{|c|c|c|}
\hline Biomarkers & Pathway & Counts \\
\hline TCF7L2 & $\begin{array}{l}\text { Wnt signaling pathway, } \\
\text { colorectal cancer, endometrial } \\
\text { cancer, basal cell carcinoma }\end{array}$ & 4 \\
\hline APC & $\begin{array}{l}\text { Wnt signaling pathway, colorectal } \\
\text { cancer, endometrial cancer, basal } \\
\text { cell carcinoma }\end{array}$ & 4 \\
\hline LEF1 & $\begin{array}{l}\text { Wnt signaling pathway, colorectal } \\
\text { cancer, endometrial cancer, B cell } \\
\text { carcinoma }\end{array}$ & 4 \\
\hline CCNE1 & $\begin{array}{l}\text { Cell cycle, oocyte meiosis, small } \\
\text { cell lung cancer }\end{array}$ & 3 \\
\hline SKP2 & Cell cycle, small cell lung cancer & 2 \\
\hline FZD7 & $\begin{array}{l}\text { Wnt signaling pathway, basal } \\
\text { cell carcinoma }\end{array}$ & 2 \\
\hline PLK1 & Cell cycle, oocyte meiosis & 2 \\
\hline BCL2 & $\begin{array}{l}\text { Colorectal cancer, small cell } \\
\text { lung cancer }\end{array}$ & 2 \\
\hline PSME4 & Proteasome & 1 \\
\hline PDSS1 & Terpenoid backbone biosynthesis & 1 \\
\hline PRKDC & Cell cycle & 1 \\
\hline TTK & Cell cycle & 1 \\
\hline MCM4 & Cell cycle & 1 \\
\hline PGR & Oocyte meiosis & 1 \\
\hline PSMA7 & Proteasome & 1 \\
\hline MDM2 & Cell cycle & 1 \\
\hline LAMB2 & Small cell lung cancer & 1 \\
\hline PSMD7 & Proteasome & 1 \\
\hline
\end{tabular}

Counts stand for the number of significant pathways. TCF7L2, transcription factor 7-like 2; $A P C$, anterior parietal cortex; $L E F 1$, lymphocyte enhancer factor-1; CCNE1, cyclin E1; SKP2, S-phase kinase-associated protein $2 ; F Z D 7$, human frizzled-7; $P L K 1$, polo-like kinase 1; BCL2, B cell lymphoma 2; PSME4, proteasome activator subunit 4; PDSS1, prenyldiphosphate synthase, subunit 1; PRKDC, promoters for human DNA-PK cs; TTK, TTK protein kinase; MCM4, minichromosome maintenance deficient 4; $P G R$, progesterone receptor; PSMA7, proteasome subunit $\alpha 7 ; M D M 2$, MDM2 proto-oncogene; $L A M B 2$, laminin subunit $\beta 2 ; P S M D 7$, proteasome 26S subunit, non-ATPase 7 .

and metastasis by regulating Wnt signalling (30,31). The regulation roles of TCF7L2 gene and related Wnt signaling pathway in breast cancer and its special subtypes have been widely confirmed (32-34). Several studies have exploited the association between the gene polymorphisms of TCF7L2 and the risk of breast cancer. Naidu et al (34) and Burwinkel et al (35) reported that TCF7L2 variants induced an increased breast cancer risk, and might be a potential candidate for the susceptibility of breast cancer. Additionally, the TCF7L2 protein is involved in the homeostasis of blood glucose and the gene polymorphisms of TCF 7L2 are identified to increase the risk of type 2 diabetes (36). Diabetes 
A

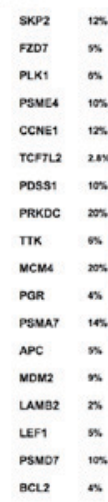

B

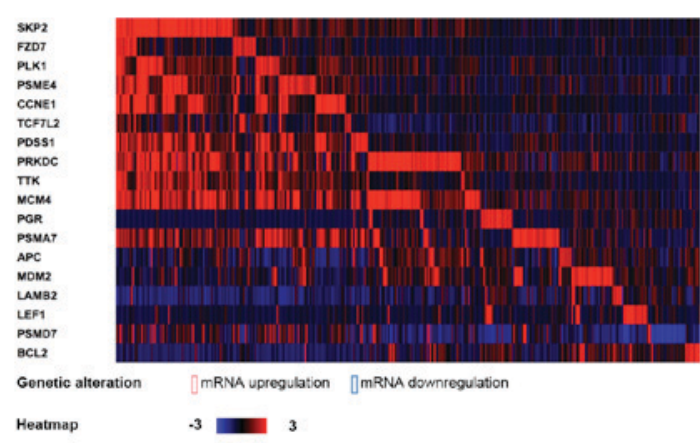

Figure 3. The annotation results and the heat map of 18 biomarkers in Luminal A breast cancer samples from the TCGA database. (A) The annotation results of 18 biomarkers in the TCGA database. Red represents upregulation and blue represents downregulation. The proportion of each gene mutation is also marked. (B) The heat map for the changes of the 18 genes in TCGA breast cancer samples. Red represents upregulation and blue represents downregulation. TCGA, The Cancer Genome Atlas; $T C F 7 L 2$, transcription factor 7-like 2; APC, anterior parietal cortex; LEF1, lymphocyte enhancer factor-1; CCNE1, cyclin E1; SKP2, S-phase kinase-associated protein 2; FZD7, human frizzled-7; PLK1, polo-like kinase 1; BCL2, B cell lymphoma 2; PSME4, proteasome activator subunit 4; PDSS1, prenyldiphosphate synthase, subunit 1; PRKDC, promoters for human DNA-PK cs; TTK, TTK protein kinase; $M C M 4$, minichromosome maintenance deficient 4; $P G R$, progesterone receptor; $P S M A 7$, proteasome subunit $\alpha 7 ; M D M 2$, MDM2 proto-oncogene; $L A M B 2$, laminin subunit $\beta 2 ; P S M D 7$, proteasome 26S subunit, non-ATPase 7.

A

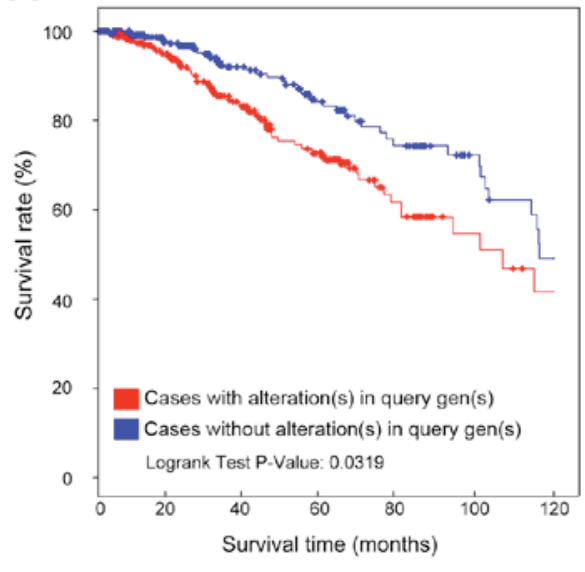

B

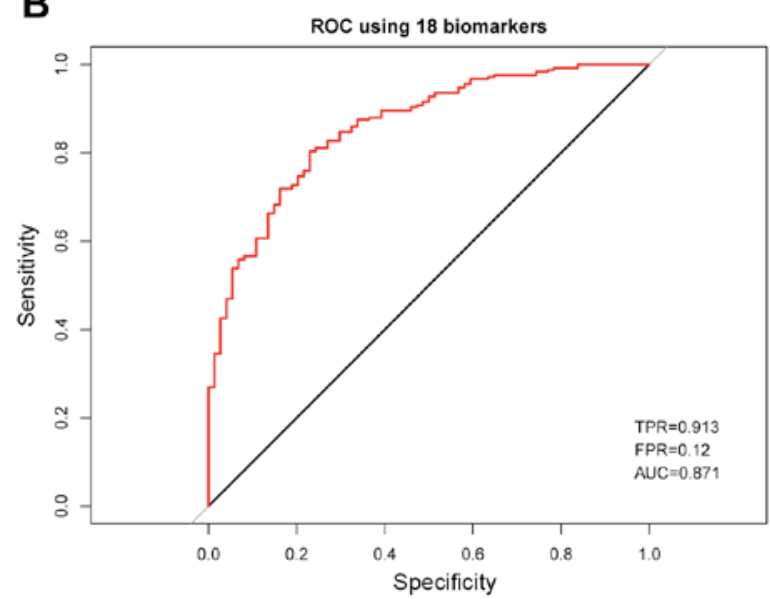

Figure 4. Survival analysis for the 18 biomarkers in train set obtained from TCGA database. (A) Survival curve for two sets of samples from TCGA database using the 18 biomarkers. The red curve represents the samples with differently expressed biomarkers, and the blue curve represents the samples with normally expressed biomarkers. (B) ROC curve for 18 biomarkers in train set. The abscissa represents sensitivity and the ordinate represents specificity. ROC, receiver operating characteristic; TGCA, The Cancer Genome Atlas; TPR, true positive rate; FPR, false positive rate; AUC, area under the curve.

A

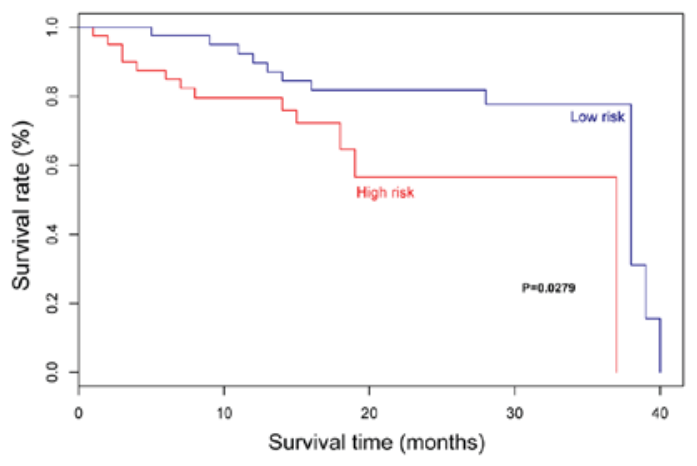

B

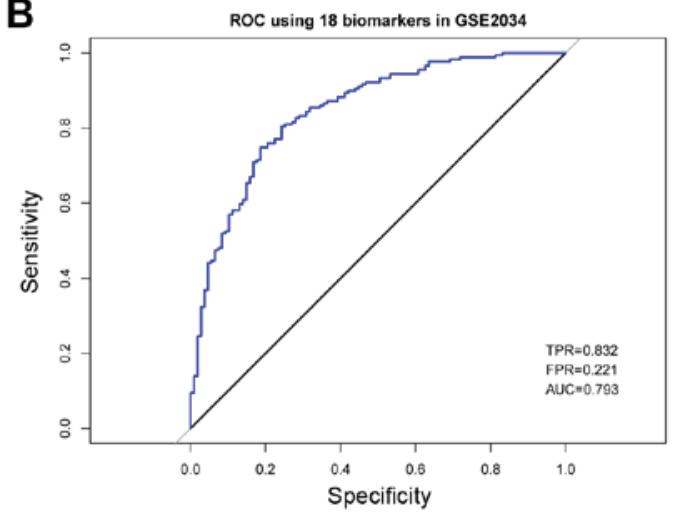

Figure 5. Survival analysis for the 18 biomarkers in test set obtained from Gene Expression Omnibus database. (A) Survival curve for the samples from the gene expression profiles of GSE2034 using the 18 biomarkers. The red curve represents samples in high risk group with differently expressed biomarkers, and the blue curve represents samples in low risk group with normally expressed biomarkers. (B) ROC curve for 18 biomarkers in test set. The abscissa represents sensitivity and the ordinate represents specificity. ROC, receiver operating characteristic; TPR, true positive rate; FPR, false positive rate; AUC, area under the curve. 
have been reported to be associated with the increased risk of breast cancer and the similar results were seen in Luminal A and B subtypes (37). Consistent with these previous studies, TCF7L2 gene was screened as a key DEG between patients with good outcomes and poor outcomes in Luminal A breast cancer.

In addition to TCF $7 L 2, A P C$ and $L E F 1$ are also involved in the above mentioned four significant pathways. The association between $A P C$ and the prognosis of breast cancer has also been reported. In a study conducted by Müller et al, the methylated APC DNA indicated the worst prognosis in breast cancer samples from the train set and the independent test set $(\mathrm{P}<0.001)$ and it was considered as an potentially independent prognostic factor for breast cancer with poor outcomes (38). The prognostic importance of $A P C$ was also been confirmed by a study which discovered that the deletion of $A P C$ was associated with a poor overall survival of breast cancer patients (39). According to the hierarchical analysis, the alterations of $A P C$ were significantly higher in ER-/PR- breast cancer compared with ER+/PR+ breast cancer samples (39). In general, patients with ER-/PR- have a worse outcome than patients with $\mathrm{ER}+/ \mathrm{PR}+$. Thus, it is reasonable to speculate that the abnormal expression of $A P C$ might be associated poor outcome in Luminal A (ER+/PR+) breast cancer. LEF1 has been widely reported to promote cancer cell metastasis, mediate chemotactic invasion, and is associated with cancer progression (40). The LEF1 overexpression has been identified as a prognostic factor for poor outcome and increased risk of liver metastasis in primary colorectal cancer (41). Delaunay et al reported that the depletion of LEF1 strongly decreased the chemotactic potential of breast cancer cells and the expression level of $L E F 1$ was associated with the risk of developing metastasis in breast cancer patients (42). As expected, the expression of LEF1 was significantly different between good and poor outcome groups and it was screened as a key DEG according to our analysis.

The 18-gene prognostic assay including the three key genes, TCF 7L2, APC and LEF1, was also demonstrated with an accurate ability to distinguish good outcomes and poor outcomes in Luminal A breast cancer. To meet the background of the precision treatment of our study would enrich the research field of specific multi-gene prognosis for breast cancer subtypes. Further study with large samples should be conducted to verify the prognostic value of this 18-gene prognostic assay and prospective study is also needed.

By conducting survival analysis, the 18-multigene assay showed effective distinguish effect on patients with different prognosis status in the low risk and high risk groups. However, the prognosis in these two groups was extraordinarily poor. The 18-gene prognostic assay should be verified in more Luminal A breast cancer samples with more typical prognosis status in further experiment.

\section{References}

1. Siegel RL, Miller KD and Jemal A: Cancer statistics, 2017. CA Cancer J Clin 67: 7-30, 2017.

2. Aure MR, Vitelli V, Jernström S, Kumar S, Krohn M, Due EU, Haukaas TH, Leivonen SK, Vollan HK, Lüders T, et al Integrative clustering reveals a novel split in the Luminal A subtype of breast cancer with impact on outcome. Breast Cancer Res 19: 44, 2017.
3. Goldhirsch A, Winer EP, Coates AS, Gelber RD, Piccart-Gebhart M, Thürlimann B and Senn HJ; Panel members: Personalizing the treatment of women with early breast cancer: Highlights of the St Gallen international expert consensus on the primary therapy of early breast cancer 2013. Ann Oncol 24: 2206-2223, 2013.

4. Jagsi R, Raad RA, Goldberg S, Sullivan T, Michaelson J, Powell SN and Taghian AG: Locoregional recurrence rates and prognostic factors for failure in node-negative patients treated with mastectomy: Implications for postmastectomy radiation. Int J Radiat Oncol Biol Phys 62: 1035-1039, 2005.

5. Carey LA, Perou CM, Livasy CA, Dressler LG, Cowan D, Conway K, Karaca G, Troester MA, Tse CK, Edmiston S, et al: Race, breast cancer subtypes, and survival in the Carolina breast cancer study. JAMA 295: 2492-2502, 2006.

6. McDermott AM, Miller N, Wall D, Martyn LM, Ball G, Sweeney KJ and Kerin MJ: Identification and validation of oncologic miRNA biomarkers for Luminal A-like breast cancer. PLoS One 9: e87032, 2014.

7. Ross JS, Hatzis C, Symmans WF, Pusztai L and Hortobágyi GN: Commercialized multigene predictors of clinical outcome for breast cancer. Oncologist 13: 477-493, 2008.

8. van 't Veer LJ, Dai H, van de Vijver MJ, He YD, Hart AA, Mao M, Peterse HL, van der Kooy K, Marton MJ, Witteveen AT, et al: Gene expression profiling predicts clinical outcome of breast cancer. Nature 415: 530-536, 2002.

9. Ma XJ, Wang Z, Ryan PD, Isakoff SJ, Barmettler A, Fuller A, Muir B, Mohapatra G, Salunga R, Tuggle JT, et al: A two-gene expression ratio predicts clinical outcome in breast cancer patients treated with tamoxifen. Cancer Cell 5: 607-616, 2004.

10. Chang JC, Wooten EC, Tsimelzon A, Hilsenbeck SG, Gutierrez MC, Elledge R, Mohsin S, Osborne CK, Chamness GC, Allred DC and O'Connell P: Gene expression profiling for the prediction of therapeutic response to docetaxel in patients with breast cancer. Lancet 362: 362-369, 2003.

11. Chang JC, Wooten EC, Tsimelzon A, Hilsenbeck SG, Gutierrez MC, Tham YL, Kalidas M, Elledge R, Mohsin S, Osborne CK, et al: Patterns of resistance and incomplete response to docetaxel by gene expression profiling in breast cancer patients. J Clin Oncol 23: 1169-1177, 2005.

12. Cleator S, Tsimelzon A, Ashworth A, Dowsett M, Dexter T, Powles T, Hilsenbeck S, Wong H, Osborne CK, O'Connell P and Chang JC: Gene expression patterns for doxorubicin (Adriamycin) and cyclophosphamide (cytoxan) (AC) response and resistance. Breast Cancer Res Treat 95: 229-233, 2006.

13. Peintinger F, Anderson K, Mazouni C, Kuerer HM, Hatzis C, Lin F, Hortobagyi GN, Symmans WF and Pusztai L: Thirty-gene pharmacogenomic test correlates with residual cancer burden after preoperative chemotherapy for breast cancer. Clin Cancer Res 13: 4078-4082, 2007.

14. Liu R, Wang X, Chen GY, Dalerba P, Gurney A, Hoey T, Sherlock G, Lewicki J, Shedden K and Clarke MF: The prognostic role of a gene signature from tumorigenic breast-cancer cells. N Engl J Med 356: 217-226, 2007.

15. Desmedt C, Piette F, Loi S, Wang Y, Lallemand F, Haibe-Kains B, Viale G, Delorenzi M, Zhang Y, d'Assignies MS, et al: Strong time dependence of the 76-gene prognostic signature for node-negative breast cancer patients in the TRANSBIG multicenter independent validation series. Clin Cancer Res 13: 3207-3214, 2007.

16. Wang Y, Klijn JG, Zhang Y, Sieuwerts AM, Look MP, Yang F, Talantov D, Timmermans M, Meijer-van Gelder ME, Yu J, et al: Gene-expression profiles to predict distant metastasis of lymph-node-negative primary breast cancer. Lancet 365 : 671-679, 2005.

17. Paik S, Shak S, Tang G, Kim C, Baker J, Cronin M, Baehner FL, Walker MG, Watson D, Park T, et al: A multigene assay to predict recurrence of tamoxifen-treated, node-negative breast cancer. $\mathrm{N}$ Engl J Med 351: 2817-2826, 2004.

18. Dowsett M, Cuzick J, Wale C, Forbes J, Mallon EA, Salter J, Quinn E, Dunbier A, Baum M, Buzdar A, et al: Prediction of risk of distant recurrence using the 21-gene recurrence score in node-negative and node-positive postmenopausal patients with breast cancer treated with anastrozole or tamoxifen: A TransATAC study. J Clin Oncol 28: 1829-1834, 2010.

19. Mamounas EP, Tang G, Fisher B, Paik S, Shak S, Costantino JP, Watson D, Geyer CE Jr, Wickerham DL and Wolmark N: Association between the 21-gene recurrence score assay and risk of locoregional recurrence in node-negative, estrogen receptor-positive breast cancer: Results from NSABP B-14 and NSABP B-20. J Clin Oncol 28: 1677-1683, 2010. 
20. Tang G, Shak S, Paik S, Anderson SJ, Costantino JP, Geyer CE Jr Mamounas EP, Wickerham DL and Wolmark N: Comparison of the prognostic and predictive utilities of the 21-gene recurrence score assay and Adjuvant! for women with node-negative, ER-positive breast cancer: Results from NSABP B-14 and NSABP B-20. Breast Cancer Res Treat 127: 133-142, 2011.

21. Albain KS, Barlow WE, Shak S, Hortobagyi GN, Livingston RB, Yeh IT, Ravdin P, Bugarini R, Baehner FL, Davidson NE, et al: Prognostic and predictive value of the 21-gene recurrence score assay in postmenopausal women with node-positive, oestrogen-receptor-positive breast cancer on chemotherapy: A retrospective analysis of a randomised trial. Lancet Oncol 11: 55-65, 2010.

22. Dowsett M, Sestak I, Lopez-Knowles E, Sidhu K, Dunbier AK, Cowens JW, Ferree S, Storhoff J, Schaper C and Cuzick J: Comparison of PAM50 risk of recurrence score with oncotype DX and IHC4 for predicting risk of distant recurrence after endocrine therapy. J Clin Oncol 31: 2783-2790, 2013.

23. Zhu X, Ying J, Wang F, Wang J and Yang H: Estrogen receptor, progesterone receptor, and human epidermal growth factor receptor 2 status in invasive breast cancer: A 3,198 cases study at National cancer center, China. Breast Cancer Res Treat 147: 551-555, 2014

24. Thomas JG, Olson JM, Tapscott SJ and Zhao LP: An efficient and robust statistical modeling approach to discover differentially expressed genes using genomic expression profiles. Genome Res 11: 1227-1236, 2001.

25. Ritchie ME, Phipson B, Wu D, Hu Y, Law CW, Shi W and Smyth GK: limma powers differential expression analyses for RNA-sequencing and microarray studies. Nucleic Acids Res 43: e47, 2015.

26. Zhang Y, Xie J, Yang J, Fennell A, Zhang C and Ma Q: QUBIC: A bioconductor package for qualitative biclustering analysis of gene co-expression data. Bioinformatics 33: 450-452, 2017.

27. Chen X, Liu L, Wang Y, Liu B, Zeng D, Jin Q, Li M, Zhang D, Liu Q and Xie H: Identification of breast cancer recurrence risk factors based on functional pathways in tumor and normal tissues. Oncotarget 8: 20679-20694, 2017.

28. Choi BY, Bair E and Lee JW: Nearest shrunken centroids via alternative genewise shrinkages. PLoS One 12: e0171068, 2017.

29. Gao J, Aksoy BA, Dogrusoz U, Dresdner G, Gross B, Sumer SO, Sun Y, Jacobsen A, Sinha R, Larsson E, et al: Integrative analysis of complex cancer genomics and clinical profiles using the cBioPortal. Sci Signal 6: pl1, 2013.

30. Ravindranath A, O'Connell A, Johnston PG and El-Tanani MK: The role of LEF/TCF factors in neoplastic transformation. Curr Mol Med 8: 38-50, 2008.

31. Reya $\mathrm{T}$ and Clevers H: Wnt signalling in stem cells and cancer. Nature 434: 843-850, 2005.

32. Dey N, Barwick BG, Moreno CS, Ordanic-Kodani M, Chen Z, Oprea-Ilies G, Tang W, Catzavelos C, Kerstann KF, Sledge GW Jr, et al: Wnt signaling in triple negative breast cancer is associated with metastasis. BMC Cancer 13: 537, 2013.
33. Vijaya Kumar A, Salem Gassar E, Spillmann D, Stock C, Sen YP, Zhang T, Van Kuppevelt TH, Hülsewig C, Koszlowski EO, Pavao MS, et al: HS3ST2 modulates breast cancer cell invasiveness via MAP kinase- and Tcf4 (Tcf712)-dependent regulation of protease and cadherin expression. Int J Cancer 135: 2579-2592, 2014.

34. Naidu R, Yip CH and Taib NA: Genetic variations in transcription factor 7-like 2 (TCF7L2) gene: Association of TCF7L2 rs12255372(G/T) or rs7903146(C/T) with breast cancer risk and clinico-pathological parameters. Med Oncol 29: 411-417, 2012.

35. Burwinkel B, Shanmugam KS, Hemminki K, Meindl A, Schmutzler RK, Sutter C, Wappenschmidt B, Kiechle M, Bartram CR and Frank B: Transcription factor 7-like 2 (TCF7L2) variant is associated with familial breast cancer risk: A case-control study. BMC Cancer 6: 268, 2006.

36. Bodhini D, Radha V, Dhar M, Narayani N and Mohan V: The rs12255372(G/T) and rs7903146(C/T) polymorphisms of the TCF7L2 gene are associated with type 2 diabetes mellitus in Asian Indians. Metabolism 56: 1174-1178, 2007.

37. Crispo A, Augustin LS, Grimaldi M, Nocerino F, Giudice A, Cavalcanti E, Di Bonito M, Botti G, De Laurentiis M, Rinaldo M, et al: Risk differences between prediabetes and diabetes according to breast cancer molecular subtypes. J Cell Physiol 232: 1144-1150, 2017.

38. Müller HM, Widschwendter A, Fiegl H, Ivarsson L, Goebel G, Perkmann E, Marth C and Widschwendter M: DNA methylation in serum of breast cancer patients: An independent prognostic marker. Cancer Res 63: 7641-7645, 2003.

39. Mukherjee N, Bhattacharya N, Alam N, Roy A, Roychoudhury S and Panda CK: Subtype-specific alterations of the Wnt signaling pathway in breast cancer: Clinical and prognostic significance. Cancer Sci 103: 210-220, 2012.

40. Wang WJ, Yao Y, Jiang LL, Hu TH, Ma JQ, Liao ZJ, Yao JT, Li DF, Wang SH and Nan KJ: Knockdown of lymphoid enhancer factor 1 inhibits colon cancer progression in vitro and in vivo. PLoS One 8: e76596, 2013.

41. Lin AY, Chua MS, Choi YL, Yeh W, Kim YH, Azzi R, Adams GA, Sainani K, van de Rijn M, So SK and Pollack JR: Comparative profiling of primary colorectal carcinomas and liver metastases identifies LEF1 as a prognostic biomarker. PLoS One 6: e16636, 2011.

42. Delaunay S, Rapino F, Tharun L, Zhou Z, Heukamp L, Termathe M, Shostak K, Klevernic I, Florin A, Desmecht H, et al: Elp3 links tRNA modification to IRES-dependent translation of LEF1 to sustain metastasis in breast cancer. J Exp Med 213: 2503-2523, 2016.

c) (i) $(-)$ This work is licensed under a Creative Commons Attribution-NonCommercial-NoDerivatives 4.0 International (CC BY-NC-ND 4.0) License. 